L1

\title{
PLATELET ALLOANTIGENS AND ALLOANTIBODIES AND THEIR CLINICAL IMPLICATIONS
}

\author{
Christian Müller-Eckhardt, M.D. \\ Institute for Clinical Immunology \& Transfusion Medicine, \\ Justus-Liebig-University, Giessen, Germany
}

Platelet alloantigens can be divided into two categories: Category I (alloantigens that platelets share with other blood or tissue cells; i.e.ABH, Lewis, Ii, P, and HLA class I) and category II, the so-called "platelet-specific" antigens. The latter comprise five biallelic systems (Zw or PlA, Ko or Sib, Bak, Yuk or Pen, Br) and a number of low frequency ("private") and high frequency ("public") antigens, i.e. Sra, $\mathrm{Va}^{\mathrm{a}}, \mathrm{Ca}$ or $\mathrm{Tu}, \mathrm{Mo}$. Their phenotype frequency varies greatly in different racial polulations. It has recently been shown that all plateletspecific antigens are due to single DNA base exchanges leading to single amino acid substitutions at the protein level. They can now be typed by either serological assays or by DNA analysis.

Immunization against these antigens can cause three distinct clinical entities: Refractoriness against platelet transfusions (PTR), neonatal alloimmune thrombocytopenia (NAIT), and post transfusion purpura (PTP).

PTR is mainly caused by HLA antibodies. The prevalence of platelet-specific antibodies in PTR has been estimated to be as high as $25 \%$, but in our experience is much lower. Anti-Zw $w^{b}$ appears to be the most frequent antibody specificity. According to a number of groups, immunization against HLA antigens can be prevented by leukocyte depletion.

NAIT is a rather rare fetomaternal incompatibility in which the mother is immunized against a paternally inherited fetal platelet antigen lacking in the mother. The most important platelet-specific 
antibodies in the caucasoid population are anti-Zwa and anti-Bra. As of Dec 31, 1992, we have studied a total of 1043 cases with suspected NAIT. Of these, 279 mothers had a platelet-specific antibody with the following specificities: anti-Zw $230(82.4 \%)$, anti-Br 39 (14\%), anti-Bak ${ }^{\mathrm{a}}$ $5(1.8 \%)$, anti-Zw $w^{b}$, anti-Bak ${ }^{b} 1$, anti-Brb 1 , anti-Sra 1,1 Isoantibody against gpIIb/IIIa from a thrombasthenic patient. Clinical problems with regard to prenatal and perinatal treatment of affected children will be discussed.

PTP is a serious adverse transfusion reaction. It almost always occurs about one week after administration of blood products containing platelet material. It is characterized by an acute bleeding tendency due to severe thrombocytopenia. Its pathogenesis is as yet obscure, but the most likely explanation is that autologous platelets are destroyed by platelet-specific alloantibodies produced in the patient which react with autologous platelets in a "pseudospecific" fashion. Experimental data supporting this view shall be presented. Of the 42 PTP cases investigated so far in our institution, the following antibody

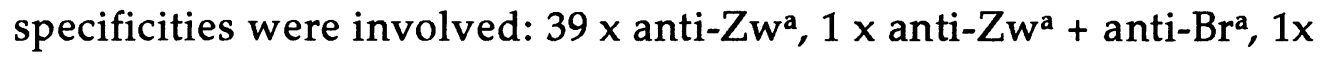
anti-Bak ${ }^{\mathrm{a}}, 1 \mathrm{x}$ anti-Bak ${ }^{\mathrm{b}}$. Treatment of choice is high-dose IgG. 